\title{
The impact of lenalidomide exposure on response and outcomes in patients with lower-risk myelodysplastic syndromes and del(5q)
}

\author{
Mikkael A. Sekeres ${ }^{1}$, Arlene S. Swern², Aristoteles Giagounidis ${ }^{3}$, Alan F. List ${ }^{4}$, Dominik Selleslag ${ }^{5}$, Moshe Mittelman ${ }^{6}$, \\ Brigitte Schlegelberger ${ }^{7}$, Gudrun Göhring ${ }^{7}$, Jack Shiansong Li ${ }^{2}$, Mary M. Sugrue ${ }^{8}$ and Pierre Fenaux ${ }^{9}$
}

\begin{abstract}
Myelodysplastic syndromes (MDS) are clonal hematopoietic malignancies that primarily affect older adults, with consequent cytopenias, blood product transfusion needs, and truncated survival ${ }^{1-3}$. Undertreatment of patients with International Prognostic Scoring System (IPSS) Low- or Intermediate (Int)-1-risk MDS and deletion $5 \mathrm{q}[\mathrm{del}(5 \mathrm{q})]$ may lead to insufficient correction of anemia, iron overload, compromised quality of life, and increased morbidity ${ }^{4,5}$. It is recommended that patients with IPSS-defined lower-risk MDS and del(5q) initiate treatment with lenalidomide at $10 \mathrm{mg} / \mathrm{day}^{6}$. Those who develop profound neutropenia or thrombocytopenia should undergo treatment interruption followed by dose reduction to manage adverse events while continuing treatment ${ }^{4,6-9}$.
\end{abstract}

It is not known whether initial lenalidomide dose (at 10 or $5 \mathrm{mg}$ ), subsequent dose reductions, or cumulative lenalidomide dose affect long-term outcomes in patients with del(5q) MDS. In this retrospective analysis, we combined data from IPSS-defined lower-risk del(5q) MDS patients treated with lenalidomide from study start in the phase 2 MDS-003 study and the phase 3 MDS-004 study to assess the impact of cumulative lenalidomide exposure on red blood cell transfusion independence (RBC-TI) $\geq$ 26 weeks, cytogenetic response, overall survival, and acute myeloid leukemia (AML)-free survival.

In the phase 2, open-label MDS-003 study $(\mathrm{NCT} 00065156)^{7}, 148$ patients received lenalidomide

\footnotetext{
Correspondence: Mikkael A. Sekeres (sekerem@ccf.org)

${ }^{1}$ Cleveland Clinic, Cleveland, OH, USA

${ }^{2}$ Celgene Corporation, Summit, NJ, USA

Full list of author information is available at the end of the article.
}

$10 \mathrm{mg}$ on days $1-21(n=46)$ or days $1-28(n=102)$ of 28-day cycles. In the phase 3, randomized, double-blind, placebo-controlled MDS-004 study (NCT00179621) ${ }^{8}, 205$ patients were centrally randomized using a validated interactive voice response system 1:1:1 to lenalidomide $10 \mathrm{mg} /$ day on days $1-21$ of 28 -day cycles $(n=69)$, or lenalidomide $5 \mathrm{mg} /$ day $(n=69)$ or placebo $(n=67)$ on days 1-28 of 28-day cycles. Key inclusion criteria for both studies included IPSS Low- or Int-1-risk del(5q) MDS with or without additional cytogenetic abnormalities, and RBC transfusion-dependent anemia. Outcomes (RBC$\mathrm{TI} \geq 26$ weeks, cytogenetic response, overall survival, and AML-free survival) were analyzed by initial lenalidomide dose group, total cumulative dose during cycles $1-3$, and incidence of dose reductions. Further details on study design can be found in the Supplementary material; full methodology and key results for these studies have been reported previously ${ }^{7,8}$.

A total of 217 patients received an initial dose of lenalidomide $10 \mathrm{mg}$ (10 mg dose group) and 69 patients received an initial dose of lenalidomide $5 \mathrm{mg}(5 \mathrm{mg}$ dose group) in the MDS-003 and MDS-004 studies. Patient baseline characteristics are shown in Supplementary Table 1; details of treatment received can be found in Supplementary Table 2 .

Overall, $\mathrm{RBC}-\mathrm{TI} \geq 26$ weeks was achieved in 148 patients (51.7\%) (Supplementary Table 3); rates of RBC$\mathrm{TI} \geq 26$ weeks were $57.1 \%$ for the $10 \mathrm{mg}$ dose group vs. $34.8 \%$ for the $5 \mathrm{mg}$ dose group $(p<0.001)$. Of 181 evaluable patients, 103 (56.9\%) achieved cytogenetic response (major or minor responses) (Supplementary Table 3): $65.2 \%$ of patients in the $10 \mathrm{mg}$ dose group vs. $30.2 \%$ in the 
Table 1 Effects of each $100 \mathrm{mg}$ cumulative lenalidomide dose increase during cycle 1 and cycles 1-3

\begin{tabular}{llr}
\hline & \multicolumn{1}{c}{$\begin{array}{l}\text { Effect of each } \mathbf{1 0 0} \text { mg increase in cumulative lenalidomide } \\
\text { dose }\end{array}$} \\
\cline { 2 - 3 } & Cycle $\mathbf{1}$ & Cycles 1-3 \\
\hline Achievement of RBC-TI $\geq 26$ weeks, OR $(95 \% \mathrm{Cl})$ & $3.41(2.19-5.32)$ & $1.59(1.37-1.85)$ \\
Achievement of cytogenetic response, OR $(95 \% \mathrm{Cl})$ & $2.61(1.50-4.56)$ & $1.31(1.11-1.56)$ \\
Progression to AML or death, HR $(95 \% \mathrm{Cl})$ & $0.62(0.48-0.80)$ & $0.85(0.78-0.92)$ \\
Death, HR $(95 \% \mathrm{Cl})$ & $0.60(0.47-0.78)$ & $0.83(0.77-0.91)$ \\
Progression to AML, HR $(95 \% \mathrm{Cl})$ & $0.72(0.48-1.09)$ & $0.98(0.86-1.11)$ \\
\hline
\end{tabular}

$A M L$ acute myeloid leukemia; $C l$ confidence interval; $H R$ hazard ratio; $O R$ odds ratio; $R B C-T I$ red blood cell transfusion independence

Table 2 Multivariate analysis of predictive factors for AML-free survival and overall survival among lenalidomidetreated patients

\begin{tabular}{|c|c|c|c|c|}
\hline \multirow[t]{2}{*}{ Baseline characteristic } & \multicolumn{2}{|c|}{ AML-free survival } & \multicolumn{2}{|l|}{ Overall survival } \\
\hline & HR $(95 \% \mathrm{Cl})$ & $p$ Value & HR $(95 \% \mathrm{Cl})$ & $p$ Value \\
\hline Dose reduction (time-varying) & $0.44(0.32-0.62)$ & $<0.0001$ & $0.47(0.33-0.66)$ & $<0.0001$ \\
\hline Total dose in cycles $1-3$, per LEN $100 \mathrm{mg}$ increase & $0.88(0.80-0.97)$ & 0.012 & $0.87(0.79-0.97)$ & 0.008 \\
\hline RBC transfusion burden, units/8 weeks & $1.08(1.03-1.13)$ & 0.0007 & $1.07(1.02-1.12)$ & 0.010 \\
\hline Log platelet count, $\times 10^{9} / /$ & $0.68(0.47-0.81)$ & 0.0007 & $0.60(0.46-0.79)$ & 0.0002 \\
\hline FAB classification (RAEB + CMML vs. RA + RARS) & $1.48(1.02-2.13)$ & 0.037 & $1.45(1.00-2.10)$ & 0.052 \\
\hline Age, per year increase & $1.04(1.02-1.05)$ & $<0.0001$ & $1.04(1.03-1.06)$ & $<0.0001$ \\
\hline
\end{tabular}

$A M L$ acute myeloid leukemia; $C I$ confidence interval; $C M M L$ chronic myelomonocytic leukemia; FAB French-American-British; $H R$ hazard ratio; $L E N$ lenalidomide; $R A$ refractory anemia; RAEB RA with excess blasts; RARS RA with ring sideroblasts; $R B C$ red blood cell

$5 \mathrm{mg}$ dose group $(p<0.001)$. Median time to onset of cytogenetic response was 148 days (range: 56-707). Of the patients who achieved a cytogenetic response, 84 of 103 (81.6\%) also achieved $\mathrm{RBC}-\mathrm{TI} \geq 26$ weeks. The odds of achieving $\mathrm{RBC}-\mathrm{TI} \geq 26$ weeks and cytogenetic response significantly increased with each $100 \mathrm{mg}$ increase in the total cumulative dose received in cycle 1 and cycles 1-3 (Table 1). A greater proportion of patients underwent dose reduction in the $10 \mathrm{mg}$ dose group vs. the $5 \mathrm{mg}$ dose group $(72.4 \%$ vs. $53.6 \% ; p<0.004)$. Patients with a dose reduction had a $79 \%$ greater chance of achieving $\mathrm{RBC}-\mathrm{TI}$ (relative risk $[R R]=1.79,95 \%$ confidence interval $[\mathrm{CI}]$ : 1.31-2.44) and a $45 \%$ greater chance of achieving a cytogenetic response $(\mathrm{RR}=1.45,95 \% \mathrm{CI}$ : 1.02-2.06) vs. patients without a dose reduction (Supplementary Table 3).

AML-free survival for patients did not significantly differ between patients in the $10 \mathrm{mg}$ dose group (median: 39.2 months, 95\% CI: 32.8-45.1) and the $5 \mathrm{mg}$ dose group (median: 44.3 months, 95\% CI: 20.1-50.8) (log-rank $p=$ $0.671)$. However, risk of progression to AML or death was reduced by $38 \%$ for each $100 \mathrm{mg}$ increase in the total cumulative dose received in cycle $1(p<0.001$; Table 1$)$. Similarly, median overall survival did not significantly differ between patients in the $10 \mathrm{mg}$ dose group (41.2 months, 95\% CI: 35.3-47.2) and the $5 \mathrm{mg}$ dose group (41.6 months, 95\% CI: 23.7-56.4) (log-rank $p=$ $0.6829)$. Risk of death was reduced by $40 \%$ for every $100 \mathrm{mg}$ increase in the total cumulative dose received in cycle $1(p<0.001$; Table 1$)$.

Both AML-free survival and overall survival were significantly longer among patients receiving a total dose of lenalidomide in cycle $1>210 \mathrm{mg}$ vs. patients receiving $\leq$ $210 \mathrm{mg}(\log$-rank $p=0.0005$ for AML-free survival, logrank $p=0.0002$ for overall survival) (Supplementary Fig. 1). Factors associated with AML-free survival and overall survival in univariate analysis are shown in Supplementary Table 4, and results of the multivariate Cox proportional hazards models analyzing covariates as predictors of AML-free survival and overall survival are presented in Table 2. In the final multivariate model, factors associated with improved AML-free survival included higher total cumulative lena-lidomide dose in cycles $1-3$ (hazard ratio $[\mathrm{HR}]=0.88,95 \% \mathrm{CI}: 0.80-0.97, p=0.012)$ and dose 
reduction $(\mathrm{HR}=0.44,95 \% \mathrm{CI}: 0.32-0.62, p<0.0001)$. Higher total cumulative lenalidomide dose in cycles $1-3$ $(\mathrm{HR}=0.87,95 \% \mathrm{CI}: 0.79-0.97, p=0.008)$ and dose reduction $(\mathrm{HR}=0.47,95 \% \mathrm{CI}: 0.33-0.66 ; p<0.0001)$ were also associated with improved overall survival.

This analysis is the largest to examine the relationship between lenalidomide exposure, dose reduction, response, and longer-term outcomes in patients with IPSS-defined lower-risk del(5q) MDS. Higher initial and cumulative lenalidomide dose in early treatment cycles was a significant predictor of improved achievement of RBC-TI $\geq$ 26 weeks and cytogenetic response, as well as improved AML-free survival and overall survival. These results suggest an induction approach of starting lenalidomide therapy at a dose of $10 \mathrm{mg}$ is associated with improved outcomes in this population of patients with del(5q) MDS.

Dose reduction was also a significant positive predictor of improved AML-free survival and overall survival in multivariate analyses. The effect of dose reduction is not independent of starting dose, as patients receiving lenalidomide $10 \mathrm{mg}$ were more likely to undergo dose reduction and experienced a better outcome than those starting on lenalidomide $5 \mathrm{mg}$. In a logistic regression carried out to determine whether patients who received dose reductions differed from those who did not, we found patients who started at lenalidomide $10 \mathrm{mg}$ were $30 \%$ more likely to receive a dose reduction than patients who started at lenalidomide $5 \mathrm{mg}$ (data not shown). Analyses of the association between dose reduction and improved survival did include those patients starting at lower doses of lenalidomide however, and retained significance in multivariable analyses. This apparent contradiction may be due to the use of lenalidomide dose reductions as part of a maintenance phase with dose reduction to $5 \mathrm{mg} /$ day, particularly in response to adverse events, which may allow patients to remain on treatment for longer, leading to increased long-term drug exposure and better outcomes. It should be noted that we analyzed the effect of dose reduction, rather than dose interruption, although patients who experienced dose reduction were likely to have also had a prior dose interruption.

Limitations of this analysis include its retrospective nature and patient population heterogeneity. Patients with less favorable disease characteristics may also have received lower doses of lenalidomide initially, potentially introducing a bias in the relationship between cumulative dose and patient outcomes, an effect possibly seen in other studies ${ }^{10}$. However, dose reductions were in fact associated with better outcomes, suggesting results are unlikely to be affected by differences in disease severity. Another potential limitation is variation in total exposure to lenalidomide between and within treatment arms, depending on their starting dose and dosing schedule. To account for this variation, outcomes were analyzed by initial lenalidomide dose group as well as total cumulative dose during early treatment. Long-term dosing beyond cycle 3 could not be evaluated due to the potential effects of dose interruptions, dose reductions, and patient crossover on later dosing in the open-label portion of the studies.

In conclusion, it is tempting, particularly in older adults, to start at lower doses of lenalidomide to avoid treatmentrelated adverse events. However, our data support the use of lenalidomide at the recommended $10 \mathrm{mg}$ dose as part of an initial induction phase of treatment. In the event of adverse events that cannot be managed through supportive mechanisms, this induction phase would be followed by a maintenance phase in which dose reductions are carried out per prescribing information guidelines ${ }^{6}$ in order to maximize exposure and treatment duration and thereby optimize outcomes in patients with lower-risk MDS and $\operatorname{del}(5 \mathrm{q})$. The association between higher early cumulative lenalidomide dose, cytogenetic response, and long-term outcomes seen in this analysis suggests lenalidomide may have a biological disease-modifying effect via targeted reduction in the del(5q) clone size.

\section{Acknowledgments \\ Celgene Corporation provided funding for this study. The authors developed the concept for this study and reviewed primary statistical data to draw conclusions from analyses. The authors received editorial support provided by James O'Reilly, PhD, from Excerpta Medica, funded by Celgene Corporation. The authors had full access to the data and are fully responsible for content and editorial decisions for this manuscript.}

\section{Author contributions}

M.A.S., A.G., A.F.L., D.S., and P.F. enrolled patients in the study, and collected and interpreted data. M.M. collected and interpreted data. G.G. and B.S. collected, interpreted, and analyzed data. A.S.S., J.S.L., and M.M.S. analyzed data. All authors contributed to drafting the report and provided final approval to submit the manuscript.

\section{Author details \\ ${ }^{1}$ Cleveland Clinic, Cleveland, OH, USA. ${ }^{2}$ Celgene Corporation, Summit, NJ, USA. ${ }^{3}$ Marien Hospital Düsseldorf, Düsseldorf, Germany. ${ }^{4}$ Moffitt Cancer Center and Research Institute, Tampa, FL, USA. ${ }^{5}$ AZ Sint-Jan Brugge-Oostende, Bruges, Belgium. ${ }^{6}$ Tel Aviv Sourasky Medical Center, Sackler Medical School, Tel Aviv University, Tel Aviv, Israel. ${ }^{7}$ Institute of Human Genetics, Hannover Medical School, Hannover, Germany. ${ }^{8}$ Formerly Celgene Corporation, Summit, NJ, USA. ${ }^{9}$ Service d'Hématologie Séniors, Hôpital Saint-Louis, Université Paris 7, Paris, France}

\section{Conflict of interest}

Dr. Sekeres has received research funding from Celgene Corporation. Dr. Swern and Dr. Li are employees of and hold equity in Celgene Corporation. Dr. Giagounidis is a consultant for and has received honoraria from Celgene Corporation. Dr. List is a consultant for and has received honoraria and research funding from Celgene Corporation. Dr. Selleslag is a consultant for and has received honoraria and research funding from Celgene Corporation, Novartis, Amgen, and GSK. Dr. Mittelman is a consultant for and has received honoraria and research funding from Celgene Corporation. Dr. Schlegelberger and Dr. Göhring have no conflicts of interest to disclose. Dr. Sugrue is a former employee of and holds equity in Celgene Corporation. Dr. Fenaux has received honoraria and research funding from Celgene Corporation. 


\section{Publisher's note}

Springer Nature remains neutral with regard to jurisdictional claims in published maps and institutional affiliations.

Supplementary Information accompanies this paper at (https://doi.org/ 10.1038/s41408-018-0126-z).

Received: 9 February 2018 Accepted: 17 August 2018

Published online: 21 September 2018

\section{References}

1. Greenberg, P. et al. International scoring system for evaluating prognosis in myelodysplastic syndromes. Blood 89, 2079-2088 (1997).

2. Sekeres, M. A. et al. Characteristics of US patients with myelodysplastic syndromes: results of six cross-sectional physician surveys. J. Natl Cancer Inst. 100, 1542-1551 (2008)

3. Greenberg, P. L. et al. Revised international prognostic scoring system (IPSS-R) for myelodysplastic syndromes. Blood 120, 2454-2465 (2012).
4. Fenaux, P. \& Adès, L. How we treat lower-risk myelodysplastic syndromes. Blood 121, 4280-4286 (2013).

5. Gerds, A. T. \& Sekeres, M. A. Initial transfusion frequency and survival in myelodysplastic syndromes: hopping onto a fast train to nowhere. Leuk. Lymphoma 55, 2221-2222 (2014).

6. Revlimid (lenalidomide) [package insert]. (Celgene Corporation, Summit, NJ, 2013).

7. List, A. et al. Lenalidomide in the myelodysplastic syndrome with chromosome 5q deletion. N. Engl. J. Med. 355, 1456-1465 (2006).

8. Fenaux, P. et al. A randomized phase 3 study of lenalidomide versus placebo in RBC transfusion-dependent patients with Low-/Intermediate-1-risk myelodysplastic syndromes with del5q. Blood 118, 3765-3776 (2011).

9. Sekeres, M. A. et al. Relationship of treatment-related cytopenias and response to lenalidomide in patients with lower-risk myelodysplastic syndromes. J. Clin. Oncol. 26, 5943-5949 (2008).

10. Sekeres, M. A. et al. Randomized phase II study of azacitidine alone or in combination with lenalidomide or with vorinostat in higher-risk myelodysplastic syndromes and chronic myelomonocytic leukemia: North American Intergroup Study SWOG S1117. J. Clin. Oncol. 35, 2745-2753 (2017). 